\title{
ON CONTRACTIONS OF CLASSICAL BASIC SUPERALGEBRAS
}

\author{
N. A. GROMOV, I. V. KOSTYAKOV, V. V. KURATOV \\ Department of Mathematics, \\ Syktyvkar Branch of IMM UrD RAS, \\ Chernova st., 3a, Syktyvkar, 167982, Russia \\ E-mail: gromov@dm.komisc.ru
}

November 6, 2018

\begin{abstract}
We define a class of orthosymplectic $\operatorname{osp}(m ; j \mid 2 n ; \omega)$ and unitary $\operatorname{sl}(m ; j \mid n ; \epsilon)$ superalgebras which may be obtained from $\operatorname{osp}(m \mid 2 n)$ and $s l(m \mid n)$ by contractions and analytic continuations in a similar way as the special linear, orthogonal and the symplectic Cayley-Klein algebras are obtained from the corresponding classical ones. Casimir operators of Cayley-Klein superalgebras are obtained from the corresponding operators of the basic superalgebras. Contractions of $s l(2 \mid 1)$ and $\operatorname{ssp}(3 \mid 2)$ are regarded as an examples.
\end{abstract}

\section{Introduction}

Since their discovery [1], [2], [3] in 1971 the supersymmetry is used in different physical theories such as Kaluza-Klein supergravity [4], supersymmetric field theories of the Wess-Zumino type [5], massless higher-spin field theories [6]. Recently the secret theory [7] (or S-theory) that includes superstring theory and its super p-brane and D-brane [8] generalizations was discussed. All these theories are build algebraically with the help of some superalgebra in their base. In this work we wish to present a wide class of Cayley-Klein 
(CK) superalgebras which may be used for constructions of different sypersymmetric models.

For an ordinary Lie groups (or algebras) the title CK was initially used for the short name of the set of a motion groups of a spaces of constant curvature. It is well known that there are $3^{n}$ n-dimensional spaces of constant curvature and their motion groups may be obtained from the orthogonal group $S O(n+1)$ with the help of contractions and analytical continuations [9]. Later the notion CK was extend to the case of unitary and symplectic groups (algebras) [10]. The typical (and attractive) property of CK groups is that all of them are depend on the same number of independent parameters as the corresponding simple classical group. On the level of Lie algebras this means that all CK algebras of the same type have the equal dimensions. A basic superalgebras include a simple algebras as an even subalgebras, so it looks quite natural to introduce a new class of superalgebras with CK algebras as an even subalgebras.

A superalgebra as an algebraic structure contain (as compared with Lie algebra) a new additional operation, namely, $Z_{2}$-grading. So under contraction of superalgebra this $Z_{2}$-grading must be conserved. To our knowledge contraction of orthosymplectic superalgebra to the superkinematics was first regarded in [11]. The detailed investigation a class of contraction of $\operatorname{sep}(1 \mid 2)$ and $\operatorname{ssp}(1 \mid 4)$ to the kinematical Poincaré and Galilei superalgebras was made in [12]. Contraction of unitary superalgebra $G s u(2)=s l(2 \mid 1)$ as well as their representations was described in [13]. Later the notion of contraction was generalized [14] to the case of Lie algebra with an arbitrary finite grading group and is known as graded contractions. Nevertheless the particular case of the simplest $Z_{2}$-grading deserve an independent interest. The preliminary results was reported in [15].

The paper is organized as follows. In section 2 the orthogonal, symplectic and special linear CK groups and algebras are briefly remind. Section 3 is devoted to the orthosymplectic CK superalgebras. CK unitary superalgebras are regarded in section 4. Casimir operators of the CK unitary and orthosymplectic superalgebras are described in section 5 . 


\section{Orthogonal, symplectic and special linear Cayley-Klein algebras}

Special linear $s l(m)$, orthogonal $s o(m)$ and symplectic $s p(2 n)$ algebras are even subalgebras of classical basic superalgebras. On the other hand all of them may be contracted and analytically continued to the set of CK algebras. Lie groups and algebras are in close relations. CK group $S O(m ; j)$ is defined as the set of transformations of vector space $\mathbf{R}_{m}(j)$, which preserve the quadratic form $x^{2}(j)=x^{t}(j) x(j)=x_{1}^{2}+\sum_{k=2}^{m}(1, k)^{2} x_{k}^{2}$, where $(i, k)=\prod_{p=\min (i, k)}^{\max (i, k)-1} j_{p},(i, i)=1$, each parameter $j_{k}=1, \iota_{k}, i$, where $\iota_{k}$ are nilpotent $\iota_{k}^{2}=0$, commutative $\iota_{k} \iota_{p}=\iota_{p} \iota_{k} \neq 0$ generators of Pimenov algebra $P(\iota)$. Cartesian components of vector $x(j) \in \mathbf{R}_{m}(j)$ are $x^{t}(j)=$ $\left(x_{1}, j_{1} x_{2}, \ldots,(1, m) x_{m}\right)^{t}$, as it is easily follows from $x^{2}(j)$. For $m \times m$ matrix $g(j) \in S O(m ; j)$ the transformation $g(j): \mathbf{R}_{m}(j) \rightarrow \mathbf{R}_{m}(j)$ means that the vector $x^{\prime}(j)=g(j) x(j)$ has exactly the same distribution of parameters $j$ among its components as $x(j)$. This requirement give an opportunity to obtain the distribution of parameters $j$ among elements of matrix $g(j)$, i.e. to build the fundamental representation of CK group $S O(m ; j)$ starting from the quadratic form. It is remarkable that the same distribution of the parameters $j$ is hold for CK Lie algebra $s o(m ; j)$, namely $A_{i k}=(i, k) a_{i k}$, for $A \in s o(m ; j)$.

The set of transformations $L(j): \mathbf{R}_{m}(j) \rightarrow \mathbf{R}_{m}(j)$ with the property $\operatorname{det} L(j)=1$ form CK special linear group $S L(m ; j)$ and the corresponding $\mathrm{CK}$ algebras $s l(m ; j)$ are given by the $m \times m$ matricies $l(j), \operatorname{tr} l(j)=0$. Let us stress that in Cartesian basis all matricies from $S L(m ; j), S O(m ; j), s l(m ; j), s o(m ; j)$ have identical distribution of parameters $j$ among its elements, i.e. they are of the same type as the matricies with elements from Pimenov algebra $P(j)$.

CK symplectic group $S p(2 n ; \omega)$ is defined as the set of transformations of $\mathbf{R}_{n}(\omega) \times \mathbf{R}_{n}(\omega)$, which preserve the bilinear form $S(\omega)=S_{1}+\sum_{k=2}^{n}[1, k]^{2} S_{k}$, where $S_{k}(y, z)=y_{k} z_{n+k}-y_{n+k} z_{k},[i, k]=\prod_{p=\min (i, k)}^{\max (i, k)-1} \omega_{k},[i, i]=1, \omega_{k}=$ $1, \xi_{k}, i, \xi_{k}^{2}=0, \xi_{k} \xi_{p}=\xi_{p} \xi_{k}$. The distribution of parameters $\omega_{k}$ among matrix elements of the fundamental representation $M(\omega)=\left(\begin{array}{cc}H(\omega) & E(\omega) \\ F(\omega) & -H^{t}(\omega)\end{array}\right)$ of the CK symplectic algebra $s p(2 n ; \omega)$ may be obtained as for orthogonal CK algebras and is as follows: $B_{i k}=[i, k] b_{i k}$, where $B=H(\omega), E(\omega), F(\omega)$. 


\section{$3 \quad$ Orthosymplectic superalgebras $\operatorname{osp}(m ; j \mid 2 n ; \omega)$}

Let $e_{I J} \in M_{m+2 n}$ satisfying $\left(e_{I J}\right)_{K L}=\delta_{I K} \delta_{J L}$ are elementary matrices. One defines the following graded matrix [16]

$$
G=\left(\begin{array}{c|cc}
I_{m} & \multicolumn{2}{|c}{0} \\
\hline 0 & 0 & I_{n} \\
& -I_{n} & 0
\end{array}\right)
$$

where $I_{m}, I_{n}$ are identity matrices. Let $i, j, \ldots=1, \ldots, m, \bar{i}, \bar{j}=m+$ $1, \ldots, m+2 n$. The generators of the orthosymplectic superalgebra $\operatorname{osp}(m \mid 2 n)$ are given by

$$
\begin{gathered}
E_{i j}=-E_{j i}=\sum_{k}\left(G_{i k} e_{k j}-G_{j k} e_{k i}\right), \quad E_{\bar{i} \bar{j}}=E_{\bar{j} \bar{i}}=\sum_{\bar{k}}\left(G_{\bar{i} \bar{k}} e_{\bar{k} \bar{j}}+G_{\bar{j} \bar{k}} e_{\bar{k} \bar{i}}\right), \\
E_{i \bar{j}}=E_{\bar{j} i}=\sum_{k} G_{i k} e_{k \bar{j}}+\sum_{\bar{k}} G_{\bar{j} \bar{k}} e_{\bar{k} i},
\end{gathered}
$$

where the even (bosonic) $E_{i j}$ generate the so(m) part, the even (bosonic) $E_{\bar{i} \bar{j}}$ generate the $\operatorname{sp}(2 n)$ part and the rest $E_{i \bar{j}}$ are the odd (fermionic) generators of superalgebra. They satisfy the following (super) commutation relations

$$
\begin{gathered}
{\left[E_{i j}, E_{k l}\right]=G_{j k} E_{i l}+G_{i l} E_{j k}-G_{i k} E_{j l}-G_{j l} E_{i k},} \\
{\left[E_{\bar{i} \bar{j}}, E_{\bar{k} \bar{l}}\right]=-G_{\bar{j} \bar{k}} E_{\bar{i} \bar{l}}-G_{\bar{i} \bar{l}} E_{\bar{j} \bar{k}}-G_{\bar{j} \bar{l}} E_{\bar{i} \bar{k}}-G_{\bar{i} \bar{k}} E_{\bar{j} \bar{l}},} \\
{\left[E_{i j}, E_{k \bar{l}}\right]=G_{j k} E_{i \bar{l}}-G_{i k} E_{j \bar{l}}, \quad\left[E_{i \bar{j}}, E_{\bar{k} \bar{l}}\right]=-G_{\bar{j} \bar{k}} E_{i \bar{l}}-G_{\bar{j} \bar{l}} E_{i \bar{k}},} \\
{\left[E_{i j}, E_{\bar{k} \bar{l}}\right]=0, \quad\left\{E_{i \bar{j}}, E_{k \bar{l}}\right\}=G_{i k} E_{\bar{j} \bar{l}}-G_{\bar{j} \bar{l}} E_{i k} .}
\end{gathered}
$$

In the matrix form $\operatorname{osp}(m \mid 2 n)=\left\{M \in M_{m+2 n} \mid M^{s t} G+G M=0\right\}$. If the matrix $M$ has the following form: $M=\sum_{i, j} a_{i j} E_{i j}+\sum_{\bar{i}, \bar{j}} b_{\overline{i j}} E_{\bar{i} \bar{j}}+\sum_{i \bar{j}} \mu_{i \bar{j}} E_{i \bar{j}}$, with $a_{i j}, b_{i \bar{j}} \in \mathbf{R}$ or $\mathbf{C}$ and $\mu_{i \bar{j}}$ as the odd nilpotent elements of Grassmann algebra: $\mu_{i \bar{j}}^{2}=0, \mu_{i \bar{j}} \mu_{i^{\prime} \bar{j}^{\prime}}=-\mu_{i^{\prime} \bar{j}^{\prime}} \mu_{i \bar{j}}$, then the corresponding supergroup $O \operatorname{sp}(m \mid 2 n)$ is obtained by the exponential map $\mathcal{M}=\exp M$ and act on (super)vector space by matrix multiplication $\mathcal{X}^{\prime}=\mathcal{M} \mathcal{X}$, where $\mathcal{X}^{t}=(x \mid \theta)^{t}$, $x$ is a $n$-dimentsional even vector and $\theta$ is a $2 m$-dimensional odd vector with odd Grassmann elements. The form inv $=\sum_{i=1}^{m} x_{i}^{2}+2 \sum_{k=1}^{n} \theta_{+k} \theta_{-k}=x^{2}+2 \theta^{2}$ is invariant under this action of orthosymplectic supergroup. 
We shall define CK orthosymplectic superalgebras starting with the invariant form

$$
i n v(j ; \omega)=u^{2} \sum_{k=1}^{m}(1, k)^{2} x_{k}^{2}+2 v^{2} \sum_{\bar{k}=m+1}^{m+n}[1, \hat{\bar{k}}]^{2} \theta_{\hat{\bar{k}}} \theta_{-\hat{\bar{k}}} \equiv u^{2} x^{2}(j)+2 v^{2} \theta^{2}(\omega),
$$

$\hat{\bar{k}}=\bar{k}-m$, when $\bar{k}=m+1, \ldots, m+n$ and $\hat{\bar{k}}=\bar{k}-m-n$, when $\bar{k}=$ $m+n+1, \ldots, m+2 n$, which is the natural unification of CK orthogonal and symplectic forms. The distributions of contraction parameters $j, \omega$ among matrix elements of the fundamental representation of $\operatorname{osp}(m ; j \mid 2 n ; \omega)$ and the transformations of the generators (2) are obtained in a standart CK manner and are as follows:

$$
E_{i k}=(i, k) E_{i k}^{*}, \quad E_{\bar{i} \bar{k}}=[\hat{\bar{i}}, \hat{\bar{k}}] E_{\bar{i} \bar{k}}^{*}, \quad E_{i \bar{k}}=u(1, i) v[1, \hat{\bar{k}}] E_{i \bar{k}}^{*},
$$

where $E^{*}$ are generators (2) of the starting superalgebra $\operatorname{osp}(m \mid 2 n)$. The transformed generators are subject of the (super) commutation relations:

$$
\begin{gathered}
{\left[E_{i j}, E_{k l}\right]=(i, j)(k, l)\left(\frac{G_{j k} E_{i l}}{(i, l)}+\frac{G_{i l} E_{j k}}{(j, k)}-\frac{G_{i k} E_{j l}}{(j, l)}-\frac{G_{j l} E_{i k}}{(i, k)}\right),} \\
{\left[E_{\bar{i} \bar{j}}, E_{\bar{k} \bar{l}}\right]=-[\hat{\bar{i}}, \hat{\bar{j}}][\hat{\bar{k}}, \hat{\bar{l}}]\left(\frac{G_{\bar{j} \bar{k}} E_{\overline{\bar{l}} \bar{l}}}{[\hat{\bar{i}}, \hat{\bar{l}}]}+\frac{G_{\bar{i} \bar{l}} E_{\bar{j} \bar{k}}}{[\hat{\bar{j}}, \hat{\bar{k}}]}+\frac{G_{\bar{i} \bar{k}} E_{\bar{j} \bar{l}}}{[\hat{\bar{j}}, \hat{\bar{l}}]}+\frac{G_{\bar{j} \bar{l}} E_{\bar{i} \bar{k}}}{[\hat{\bar{i}}, \hat{\bar{k}}]}\right),} \\
{\left[E_{i j}, E_{\bar{k} \bar{l}}\right]=0, \quad\left[E_{i j}, E_{k \bar{l}}\right]=(i, j)(1, k)\left(\frac{G_{j k} E_{i \bar{l}}}{(1, i)}-\frac{G_{i k} E_{j \bar{l}}}{(1, j)}\right),} \\
{\left[E_{i \bar{j}}, E_{\bar{k} \bar{l}}\right]=-[1, \hat{\bar{j}}][\hat{\bar{k}}, \hat{\bar{l}}]\left(\frac{G_{\bar{j} \bar{k}} E_{i \bar{l}}}{[1, \hat{\bar{l}]}}+\frac{G_{\bar{j} \bar{l}} E_{i \bar{k}}}{[1, \hat{\bar{k}}]}\right)} \\
\left\{E_{i \bar{j}}, E_{k \bar{l}}\right\}=u^{2} v^{2}(1, i)[1, \hat{\bar{j}}](1, k)[1, \hat{\bar{l}}]\left(\frac{G_{i k} E_{\bar{j} \bar{l}}}{[\hat{\bar{j}}, \hat{\bar{l}}]}-\frac{G_{\bar{j} \bar{l}} E_{i k}}{(i, k)}\right) .
\end{gathered}
$$

For $u=\iota$ or $v=\iota, \iota^{2}=0$ superalgebra $\operatorname{osp}(m \mid 2 n)$ is contracted to inhomogeneous superalgebra, which is semidirect sum $\left\{E_{i \bar{j}}\right\} \boxplus(s o(m) \oplus s p(2 n))$, with all anticommutators of the odd generators equal to zero $\left\{E_{i \bar{j}}, E_{k \bar{p}}\right\}=0$. 


\subsection{Example: CK contractions of $\operatorname{ssp}(3 \mid 2)$}

This superalgebra has $s o(3)$ as even subalgebra therefore their contractions to the kinematical $(1+1)$ Poincare, Newton and Galilei superalgebras may be fulfilled according to general CK scheme of the first section. But unlike of two odd generators of $\operatorname{osp}(1 \mid 2)$ the superalgebra $\operatorname{osp}(3 \mid 2)$ has six odd gen-

erators. In the basis $X_{i k}=E_{k i}, k, i=1,2,3, F=\frac{1}{2} E_{44}, E=-\frac{1}{2} E_{55}, H=$ $-E_{45}, Q_{k}=E_{k 4}, Q_{-k}=E_{k 5}$ the generators are affected by the contraction coefficients $j_{1}, j_{2}$ in the following way

$$
X_{i k} \rightarrow(i, k) X_{i k}, \quad Q_{ \pm k} \rightarrow(1, k) Q_{ \pm k}
$$

and $H, F, E$ are remained unchanged. Then superalgebra $o s p(3 ; j \mid 2)$ is given by

$$
\begin{gathered}
{\left[X_{12}, X_{13}\right]=j_{1}^{2} X_{23}, \quad\left[X_{13}, X_{23}\right]=j_{2}^{2} X_{12}, \quad\left[X_{23}, X_{12}\right]=X_{13}} \\
{[H, E]=2 E, \quad[H, F]=-2 F, \quad[E, F]=H} \\
{\left[X_{i k}, Q_{ \pm i}\right]=Q_{ \pm k}, \quad\left[X_{i k}, Q_{ \pm k}\right]=-(i, k)^{2} Q_{ \pm i}^{2}, \quad i<k} \\
{\left[H, Q_{ \pm k}\right]=\mp Q_{ \pm k}, \quad\left[E, Q_{k}\right]=-Q_{-k}, \quad\left[F, Q_{-k}\right]=-Q_{k}} \\
\left\{Q_{k}, Q_{k}\right\}=(1, k)^{2} F, \quad\left\{Q_{-k}, Q_{-k}\right\}=-(1, k)^{2} E \\
\left\{Q_{k}, Q_{-k}\right\}=-(1, k)^{2} H, \quad\left\{Q_{ \pm i}, Q_{\mp k}\right\}= \pm(1, k)^{2} X_{i k} .
\end{gathered}
$$

The non-minimal Poincare superalgebra is obtained for $j_{1}=\iota_{1}, j_{2}=i$ and has the structure of the semidirect sum $T \boxplus\left(\left\{X_{23}\right\} \oplus \operatorname{osp}(1 \mid 2)\right)$, where abelian $T=\left\{X_{12}, X_{13}, Q_{ \pm 2}, Q_{ \pm 3}\right\}$ and $\operatorname{osp}(1 \mid 2)=\left\{H, E, F, Q_{ \pm 1}\right\}$. The Newton superalgebra $\operatorname{osp}\left(3 ; \iota_{2} \mid 2\right)=T_{2} Ð \operatorname{osp}(2 \mid 2)$, where $T_{2}=\left\{X_{13}, X_{23}, Q_{ \pm 3}\right\}$ and $\operatorname{ssp}(2 \mid 2)$ is generated by $X_{12}, H, E, F, Q_{ \pm 1}, Q_{ \pm 2}$. Finally the non-minimal Galilei superalgebra may be presented as semidirect sums $\operatorname{osp}\left(3 ; \iota_{1}, \iota_{2} \mid 2\right)=$ $\left(T \boxplus\left\{X_{23}\right\}\right) \boxplus \operatorname{osp}(1 \mid 2)=T \boxplus\left(\left\{X_{23}\right\} \oplus \operatorname{osp}(1 \mid 2)\right)$.

\section{$4 \quad$ Unitary superalgebras $\operatorname{sl}(m ; j \mid n ; \epsilon)$}

The superalgebras $s l(m \mid n)$ can be generated as a matrix superalgebras by taking matrices of the form [16]

$$
M=\left(\begin{array}{cc}
X_{m m} & T_{m n} \\
T_{n m} & X_{n n}
\end{array}\right)
$$


where $X_{m m}$ and $X_{n n}$ are $g l(m)$ and $g l(n)$ matrices, $T_{m n}$ and $T_{n m}$ are $m \times n$ and $n \times m$ matrices respectively, with the supertrace condition

$$
\operatorname{str}(M)=\operatorname{tr}\left(X_{m m}\right)-\operatorname{tr}\left(X_{n n}\right)=0 .
$$

This matrix superalgebra is the set of transformations of the superspace with $m$ even coordinates $x_{1}, \ldots, x_{m}$ and $n$ odd ones $\theta_{1}, \ldots, \theta_{n}$.

A basis of superalgebra $s l(m \mid n)$ can be constructed as follows. Define the $(m+n)^{2}-1$ generators

$$
\begin{array}{ll}
E_{i j}=e_{i j}-\frac{1}{m-n} \delta_{i j}\left(\sum_{k=1}^{m} e_{k k}+\sum_{\bar{k}=m+1}^{m+n} e_{\bar{k} \bar{k}}\right), & E_{i \bar{j}}=e_{i \bar{j}}, \\
E_{\bar{i} \bar{j}}=e_{\bar{i} \bar{j}}+\frac{1}{m-n} \delta_{\bar{i} \bar{j}}\left(\sum_{k=1}^{m} e_{k k}+\sum_{\bar{k}=m+1}^{m+n} e_{\bar{k} \bar{k}}\right), & E_{\bar{i} j}=e_{\bar{i} j},
\end{array}
$$

where the indices $i, j, \ldots$ run from 1 to $m$ and $\bar{i}, \bar{j}, \ldots$ from $m+1$ to $m+n$. The generators of $\operatorname{sl}(m \mid n)$ in the Cartan-Weyl basis are given by

$$
\begin{aligned}
H_{i} & =E_{i j}-E_{i+1, j+1}, \quad 1 \leq i \leq m-1, \\
H_{\bar{i}} & =E_{\overline{i i}}-E_{\bar{i}+1, \bar{i}+1}, \quad m+1 \leq \bar{i} \leq m+n-1, \\
H_{m} & =E_{m m}+E_{m+1, m+1}, \\
E_{i j} & \text { for } \operatorname{sl}(m), \quad E_{\overline{i j}} \quad \text { for } \operatorname{sl}(n), \\
E_{i \bar{j}} & \text { and } \quad E_{\bar{i} j} \quad \text { for the odd part }
\end{aligned}
$$

and their commutation relations looked as

$$
\begin{aligned}
& {\left[H_{I}, H_{J}\right]=0} \\
& {\left[H_{K}, E_{I J}\right]=\delta_{I K} E_{K J}-\delta_{I, K+1} E_{K+1, J}-\delta_{K J} E_{I K}+\delta_{K+1, J} E_{I, K+1},(K \neq m),} \\
& {\left[H_{m}, E_{I J}\right]=\delta_{I m} E_{m J}-\delta_{I, m+1} E_{m+1, J}-\delta_{m J} E_{I m}+\delta_{m+1, J} E_{I, m+1} \text {, }} \\
& {\left[E_{I J}, E_{K L}\right]=\delta_{J K} E_{I L}-\delta_{I L} E_{K J} \text { for } E_{I J} \text { and } E_{K L} \text { even, }} \\
& {\left[E_{I J}, E_{K L}\right]=\delta_{J K} E_{I L}-\delta_{I L} E_{K J} \text { for } E_{I J} \text { even and } E_{K L} \text { odd, }} \\
& \left\{E_{I J}, E_{K L}\right\}=\delta_{J K} E_{I L}+\delta_{I L} E_{K J} \quad \text { for } E_{I J} \text { and } E_{K L} \text { odd. }
\end{aligned}
$$

CK special linear (or unitary) superalgebras $s l(m ; j \mid n ; \epsilon)$ are consistent with the transformations of (super) vectors

$$
\mathcal{X}^{t}(j, \epsilon)=\left(x_{1}, j_{1} x_{2}, \ldots,(1, m) x_{m} \mid \nu\left(x_{m+1}, \epsilon_{1} x_{m+2}, \ldots,[1, n] x_{m+n}\right)\right)^{t},
$$


where the odd components are denote as $x_{m+1}=\theta_{1}, \ldots, x_{m+n}=\theta_{n}$ and $\hat{\bar{i}}=\bar{i}-m, \hat{\bar{k}}=\bar{k}-m=1, \ldots, n,[\hat{\bar{i}}, \hat{\bar{k}}]=\prod_{l=\min (\hat{\bar{i}}, \hat{\bar{k}})}^{\max (\hat{\overline{\hat{k}}})-1} \epsilon_{l}, \epsilon_{l}=1, \xi_{l}, i, \xi_{l}^{2}=$ $0, \xi_{l} \xi_{p}=\xi_{p} \xi_{l} \neq 0$. The components of $\mathcal{X}(j ; \epsilon)$ are choosen in such a way that the contraction parameters $\epsilon_{l}$ of the odd components were independent of the contraction parameters $j_{l}$ of the even ones. The transformations of the standart generators (12) (marked with star) of the special linear superalgebra $s l(m \mid n)$ to the generators of $\operatorname{sl}(m ; j \mid n, \epsilon)$ are given by

$$
\begin{gathered}
H_{I}=H_{I}^{*}, E_{i j}=(i, j) E_{i j}^{*}, E_{\overline{i j}}=[\hat{\bar{i}}, \hat{\bar{j}}] E_{\bar{i} \bar{j}}^{*}, i \neq j, \bar{i} \neq \bar{j}, \\
E_{i \bar{j}}=\nu(1, i)[1, \hat{\bar{j}}] E_{i \bar{j}}^{*}, \quad E_{\bar{i} j}=\nu(1, j)[1, \hat{\bar{i}}] E_{\bar{i} j}^{*} .
\end{gathered}
$$

Nonzero commutators and anticommutators are easily obtained from the corresponding commutation relations (13) of the initial superalgebra $\operatorname{sl}(m \mid n)$ in the form

$$
\begin{aligned}
& {\left[H_{K}, E_{I J}\right]=\delta_{I K} E_{K J}-\delta_{I, K+1} E_{K+1, J}-\delta_{K J} E_{I K}+\delta_{K+1, J} E_{I, K+1} \text {, }} \\
& {\left[E_{i j}, E_{j l}\right]= \begin{cases}E_{i l}, & i<j<l, l<j<i, l \neq i, \\
(l, j)^{2} E_{i l}, & i<l<j \text { or } j<l<i \\
(i, j)^{2} E_{i l}, & l<i<j \text { or } j<i<i\end{cases} } \\
& {\left[E_{i j}, E_{k j}\right]= \begin{cases}-E_{k j}, & k<i<j, j<i<k, k \neq j \\
-(i, j)^{2} E_{k j}, & i<j<k \text { or } k<j<i \\
-(i, k)^{2} E_{k j}, & i<k<j \text { or } j<k<i\end{cases} } \\
& {\left[E_{i j}, E_{j i}\right]=(i, j)^{2}\left(E_{i i}-E_{j j}\right),} \\
& {\left[E_{\bar{i} \bar{j}}, E_{\bar{j} \bar{l}}\right]= \begin{cases}E_{\bar{i}}, & \bar{i}<\bar{j}<\bar{l}, \bar{l}<\bar{j}<\bar{i}, \bar{l} \neq \bar{i} \\
{[\hat{\bar{l}}, \hat{j}]^{2} E_{\bar{i} \bar{l}},} & \bar{i}<\bar{l}<\bar{j} \text { or } \bar{j}<\bar{l}<\bar{i} \\
{\left[\hat{\bar{i}}, \hat{\bar{j}}^{2}\right]_{\bar{i} \bar{l}},} & \bar{l}<\bar{i}<\bar{j} \text { or } \bar{j}<\bar{i}<\bar{k}\end{cases} } \\
& {\left[E_{\bar{i} \bar{j}}, E_{\bar{k} \bar{j}}\right]= \begin{cases}-E_{\bar{k} \bar{j}}, & \bar{k}<\bar{i}<\bar{j}, \bar{j}<\bar{i}<\bar{k}, \bar{k} \neq \bar{j} \\
-[\hat{\bar{i}}, \bar{j}]^{2} E_{\bar{k} \bar{j}}, & \bar{i}<\bar{j}<\bar{k} \text { or } \bar{k}<\bar{j}<\bar{i} \\
-[\hat{\bar{i}}, \hat{\bar{k}}]^{2} E_{\bar{k} \bar{j}}, & \bar{i}<\bar{k}<\bar{j} \text { or } \bar{j}<\bar{k}<\bar{i}\end{cases} } \\
& {\left[E_{\bar{i} \bar{j}}, E_{\bar{j} \bar{i}}\right]=[\hat{\bar{i}}, \hat{\bar{j}}]^{2}\left(E_{\bar{i} i}-E_{\bar{j} \bar{j}}\right) \text {, }} \\
& {\left[E_{i j}, E_{j \bar{l}}\right]=\left\{\begin{array}{ll}
(i, j)^{2} E_{i \bar{l}}, & i<j, \\
E_{i \bar{l}}, & i>j,
\end{array} \quad\left[E_{i j}, E_{\bar{k} i}\right]=\left\{\begin{array}{ll}
-E_{\bar{k} j}, & i<j, \\
-(j, i)^{2} E_{\bar{k} j}, & i>j,
\end{array},\right.\right.}
\end{aligned}
$$




$$
\begin{gathered}
{\left[E_{\bar{i} \bar{j}}, E_{k \bar{i}}\right]=\left\{\begin{array}{ll}
-E_{k \bar{j}}, & \bar{i}<\bar{j}, \\
-[\hat{\bar{j}}, \hat{\bar{j}}]^{2} E_{k \bar{j}}, & \bar{i}>\bar{j},
\end{array} \quad\left[E_{\bar{i} \bar{j}}, E_{\bar{j} l}\right]= \begin{cases}{[\hat{\bar{i}}, \hat{\bar{j}}]^{2} E_{\bar{i} l},} & \bar{i}<\bar{j}, \\
E_{\bar{i} l}, & \bar{i}>\bar{j},\end{cases} \right.} \\
\left\{E_{i \bar{j}}, E_{\bar{j} l}\right\}= \begin{cases}\nu^{2}[1, \hat{\bar{j}}]^{2}(1, i)^{2} E_{i l}, & i<l, \\
\nu^{2}[1, \hat{\bar{j}}]^{2}(1, l)^{2} E_{i l}, & i>l,\end{cases} \\
\left\{E_{i \bar{j}}, E_{\bar{k} i}\right\}= \begin{cases}\nu^{2}(1, i)^{2}[1, \hat{\bar{j}}]^{2} E_{\bar{k} \bar{j}}, & \bar{j}<\bar{k}, \\
\nu^{2}(1, i)^{2}[1, \hat{\bar{k}}]^{2} E_{\bar{k} \bar{j}}, & \bar{j}>\bar{k},\end{cases} \\
\left\{E_{i \bar{j}}, E_{\bar{j} i}\right\}=\nu^{2}(1, i)^{2}[1, \hat{\bar{j}}]^{2}\left(E_{i i}+E_{\bar{j} \bar{j}}\right) .
\end{gathered}
$$

For $\nu=\iota$ superalgebra $\operatorname{sl}(m \mid n)$ is contracted to inhomogeneous superalgebra, which is semidirect sum $\left\{E_{i \bar{j}}, E_{\overline{i j}}\right\} \boxplus(\operatorname{sl}(m) \bigoplus \operatorname{sl}(n))$, with all anticommutators of the odd generators equal to zero.

\subsection{Example: CK contractions of $\operatorname{sl}(2 \mid 1)$}

The generators of superalgebra $\operatorname{sl}\left(2 ; j_{1} ; \nu \mid 1\right)$ is given by [16]

$$
\begin{aligned}
& H=\left(\begin{array}{cc|c}
\frac{1}{2} & 0 & 0 \\
0 & -\frac{1}{2} & 0 \\
\hline 0 & 0 & 0
\end{array}\right), \quad Z=\left(\begin{array}{cc|c}
\frac{1}{2} & 0 & 0 \\
0 & \frac{1}{2} & 0 \\
\hline 0 & 0 & 1
\end{array}\right) \\
& E_{12}=E^{+}=\left(\begin{array}{cc|c}
0 & j_{1} & 0 \\
0 & 0 & 0 \\
\hline 0 & 0 & 0
\end{array}\right), \quad E_{21}=E^{-}=\left(\begin{array}{cc|c}
0 & 0 & 0 \\
j_{1} & 0 & 0 \\
\hline 0 & 0 & 0
\end{array}\right) \text {, } \\
& E_{13}=\bar{F}^{+}=\left(\begin{array}{cc|c}
0 & 0 & \nu \\
0 & 0 & 0 \\
\hline 0 & 0 & 0
\end{array}\right), E_{31}=F^{-}=\left(\begin{array}{cc|c}
0 & 0 & 0 \\
0 & 0 & 0 \\
\hline \nu & 0 & 0
\end{array}\right) \text {, } \\
& E_{32}=F^{+}=\left(\begin{array}{cc|c}
0 & 0 & 0 \\
0 & 0 & 0 \\
\hline 0 & \nu j_{1} & 0
\end{array}\right), E_{23}=\bar{F}^{-}=\left(\begin{array}{cc|c}
0 & 0 & 0 \\
0 & 0 & \nu j_{1} \\
\hline 0 & 0 & 0
\end{array}\right)
\end{aligned}
$$

and acts on the superspace $\left(x_{1}, j_{1} x_{2} \mid \nu \theta_{1}\right)$. The commutation relations are represented as

$$
\left[H, E^{ \pm}\right]= \pm E^{ \pm},\left[E^{+}, E^{-}\right]=2 j_{1}^{2} H,[Z, H]=\left[Z, E^{ \pm}\right]=\left[E^{ \pm}, \bar{F}^{ \pm}\right]=\left[E^{ \pm}, F^{ \pm}\right]=0
$$




$$
\begin{gathered}
{\left[H, \bar{F}^{ \pm}\right]= \pm \frac{1}{2} \bar{F}^{ \pm},\left[H, F^{ \pm}\right]= \pm \frac{1}{2} F^{ \pm},\left[Z, F^{ \pm}\right]=\frac{1}{2} F^{ \pm},\left[Z, \bar{F}^{ \pm}\right]=-\frac{1}{2} \bar{F}^{ \pm},} \\
{\left[E^{+}, F^{-}\right]=-F^{+},\left[E^{-}, F^{+}\right]=-j_{1}^{2} F^{-},\left[E^{+}, \bar{F}^{-}\right]=j_{1}^{2} \bar{F}^{+},\left[E^{-}, \bar{F}^{+}\right]=\bar{F}^{-},} \\
\left\{F^{+}, \bar{F}^{-}\right\}=\nu^{2} j_{1}^{2}(Z-H), \quad\left\{F^{-}, \bar{F}^{+}\right\}=\nu^{2}(Z+H), \\
\left\{\bar{F}^{+}, F^{+}\right\}=\nu^{2} E^{+},\left\{\bar{F}^{-}, F^{-}\right\}=\nu^{2} E^{-},\left\{\bar{F}^{+}, \bar{F}^{-}\right\}=\left\{F^{+}, F^{-}\right\}=0 .
\end{gathered}
$$

For $\nu=\iota$ we obtain the semidirect sum of the abelian odd subalgebra with the direct sum of the even subalgebras, namely, $\operatorname{sl}\left(2 ; j_{1} ; \iota \mid 1\right)=\left\{F^{ \pm}, \bar{F}^{ \pm}\right\} \boxplus$ $(u(1) \oplus s l(2))$. Two-dimensional contraction $\nu=\iota, j_{1}=\iota_{1}$ give in result similar semidirect sum $s l\left(2 ; \iota_{1} ; \iota \mid 1\right)=\left\{F^{ \pm}, \bar{F}^{ \pm}\right\} \boxplus\left(u(1) \oplus \operatorname{sl}\left(2 ; \iota_{1}\right)\right)$ but with the subalgebra $s l\left(2 ; \iota_{1}\right)=\left\{H, E^{ \pm}\right\}$instead of $s l(2)$. Under contraction $j_{1}=\iota_{1}$ we have the semidirect sum $\operatorname{sl}\left(2 ; \iota_{1} ; \nu \mid 1\right)=\left\{E^{ \pm}, F^{+}, \bar{F}^{-}\right\} \boxplus\left\{H, Z, F^{-}, \bar{F}^{+}\right\}$of the subsuperalgebras each of them generated both even and odd generators.

\section{Casimir operators}

The study of Casimir operators plays a grate role in the representation theory of simple Lie algebras since their eigenvalues characterize a representations. In the case of Lie superalgebras their eigenvalues completely characterize a typical representation while they are identically vanishing on an atypical representation. An element $C$ of universal enveloping superalgebra $U(A)$ commuting with all elements of $U(A)$ is called a Casimir operator of superalgebra $A$. The algebra of the Casimir operators of $A$ is the $Z_{2}$-center of $U(A)$.

Casimir operators of the basic Lie superalgebras can be constructed as follows [16], [17], [18]. Let $A=\operatorname{sl}(m \mid n)$ with $m \neq n$ or $\operatorname{osp}(m \mid n)$ be a basic Lie superalgebra. Let $\left\{E_{I J}\right\}$ be a matrix basis of generators of $A$ where $I, J=1, \ldots, m+n$ with $\operatorname{deg} I=0$ for $I=1, \ldots, m$ and $\operatorname{deg} I=1$ for $I=m+1, \ldots, m+n$. Then defining $(\bar{E})_{I K}=(-1)^{\operatorname{deg} K} E_{I K}$, a standard sequence of Casimir operators is given by

$$
\begin{gathered}
C_{p}=\operatorname{str}\left(\bar{E}^{p}\right)=\sum_{I=1}^{m+n}(-1)^{\operatorname{deg} I}\left(\bar{E}^{p}\right)_{I I}= \\
=\sum_{I, I_{1}, \ldots, I_{p-1}=1}^{m+n} E_{I I_{1}}(-1)^{\operatorname{deg} I_{1}} \ldots E_{I_{k} I_{k+1}}(-1)^{\operatorname{deg} I_{k+1}} \ldots E_{I_{p-1} I} .
\end{gathered}
$$


In the case of $\operatorname{sl}(m \mid n)$ with $m \neq n$ one finds for example $C_{1}=0$ and

$$
C_{2}=\sum_{i, j=1}^{m} E_{i j} E_{j i}-\sum_{\bar{k}, \bar{l}=m+1}^{m+n} E_{\bar{k} \bar{l}} E_{\bar{l} \bar{k}}+\sum_{i=1}^{m} \sum_{\bar{k}=m+1}^{m+n}\left(E_{\bar{k} i} E_{i \bar{k}}-E_{i \bar{k}} E_{\bar{k} i}\right)-\frac{m-n}{m n} Y^{2} .
$$

The diagonal elements of matrix $\bar{E}$ are taken in the form $(\bar{E})_{i i}=E_{i i}+$ $\frac{1}{m} Y,(\bar{E})_{\bar{k} \bar{k}}=-E_{\bar{k} \bar{k}}+\frac{1}{n} Y$ and two conditions on generators: $\sum_{i=1}^{m} E_{i i}=$ $0, \sum_{\bar{k}=m+1}^{m+n} E_{\bar{k} \bar{k}}=0$ are taken into consideration. In the case of $\operatorname{osp}(m \mid n)$ one finds $C_{1}=0$ and

$$
C_{2}=\sum_{i, j=1}^{m} E_{i j} E_{j i}-\sum_{\bar{k}, \bar{l}=m+1}^{m+n} E_{\bar{k} \bar{l}} E_{\overline{l k}}+\sum_{i=1}^{m} \sum_{\bar{k}=m+1}^{m+n}\left(E_{\bar{k} i} E_{i \bar{k}}-E_{i \bar{k}} E_{\bar{k} i}\right) .
$$

One has to stress that unlike the algebraic case, the center of $U(A)$ for the classical Lie superalgebras is in general not finitely generated. For only Lie superalgebra $\operatorname{ssp}(1 \mid 2 n)$ the center of its universal enveloping superalgebra is generated by $n$ Casimir operators of degree $2,4, \ldots, 2 n$.

To obtain Casimir operators of superalgebra $\operatorname{sl}(m ; j \mid n ; \epsilon)$ we shall proceed in the standart manner. First we get the matrix $\bar{E}(j ; \epsilon)$. For this we put in matrix $\bar{E}$ the new generators of $s l(m ; j \mid n ; \epsilon)$ instead of the old ones of $\operatorname{sl}(m ; n)$ according to (15) and denote the obtained matrix as $\bar{E}(\rightarrow)$. In general its elements are undefined for nilpotent values of parameters $j, \epsilon, \nu$. So it is necessary to multiply $\bar{E}(\rightarrow)$ on minimal multiplier which eliminate all undefined expressions in matrix elements, namely, $\nu(1, m)[1, n]$. Finally we have

$$
\bar{E}(j ; \epsilon)=\nu(1, m)[1, n] \bar{E}(\rightarrow)
$$

with matrix elements $(k \neq p, \bar{k} \neq \bar{p})$

$$
\begin{array}{cl}
(\bar{E}(j ; \epsilon))_{k k}=\nu(1, m)[1, n]\left(E_{k k}+\frac{1}{m} Y\right), & (\bar{E}(j ; \epsilon))_{\bar{k} \bar{k}}=\nu(1, m)[1, n]\left(-E_{\bar{k} \bar{k}}+\frac{1}{n} Y\right), \\
(\bar{E}(j ; \epsilon))_{k p}=\nu(1, k)(p, m)[1, n] E_{k p}, \quad(\bar{E}(j ; \epsilon))_{\bar{k} \bar{p}}=\nu(1, m)[1, \hat{\bar{k}}][\hat{\bar{p}}, n] E_{\bar{k} \bar{p}}, \\
(\bar{E}(j ; \epsilon))_{i \bar{k}}=-(i, m)[\hat{\bar{k}}, n] E_{i \bar{k}}, \quad(\bar{E}(j ; \epsilon))_{\bar{i} k}=(k, m)[\hat{\bar{i}}, n] E_{\bar{i} k} .
\end{array}
$$

Maximal multiplier $\nu(1, m)[1, n]$ have the diagonal elements and minimal unit multiplier have the matrix elements $(\bar{E}(j ; \epsilon))_{m, m+n}=E_{m, m+n},(\bar{E}(j ; \epsilon))_{m+n, m}=$ $E_{m+n, m}$. 
The sequence of Casimir operators of $\operatorname{sl}(m ; j \mid n ; \epsilon)$ is given by

$$
C_{p}(j ; \epsilon)=\operatorname{str} \bar{E}^{p}(j ; \epsilon)=\nu^{p}(1, m)^{p}[1, n]^{p} \operatorname{str}(\bar{E}(\rightarrow))^{p} .
$$

Indeed, let $X^{\star}$ be an arbitrary generator of $s l(m \mid n)$. Under computing $\left[C_{p}, X^{\star}\right]=0$ we get identical terms but with different signs (plus and minus) so their sum is equal to zero. Under transformation of this commutator to the corresponding commutator of $s l(m ; j \mid n ; \epsilon)$ identical terms are multiplied on identical multipliers therefore their sum remains equal to zero, i.e. $\left[C_{p}(j ; \epsilon), X\right]=0$.

Let us illustrate the above expressions on the simple example of $s l\left(2 ; j_{1} \mid 1\right)$ superalgebra. The generators are transformed as follows

$$
\begin{gathered}
E_{11}=E_{11}^{\star}, Y=Y^{\star}, E_{12}=j_{1} E_{12}^{\star}, E_{21}=j_{1} E_{21}^{\star}, E_{13}=\nu E_{13}^{\star}, \\
E_{31}=\nu E_{31}^{\star}, E_{23}=\nu j_{1} E_{23}^{\star}, E_{32}=\nu j_{1} E_{32}^{\star} .
\end{gathered}
$$

and matrix $\bar{E}\left(j_{1}\right)$ according to (22),(23) is given by

$$
\begin{aligned}
\bar{E}\left(j_{1}\right) & =\nu j_{1} \bar{E}(\rightarrow)=\nu j_{1}\left(\begin{array}{cc|c}
E_{11}+\frac{1}{2} Y & \frac{1}{j_{1}} E_{12} & -\frac{1}{\nu} E_{13} \\
\frac{1}{j_{1}} E_{21} & -E_{22}+\frac{1}{2} Y & -\frac{1}{\nu j_{1}} E_{23} \\
\hline \frac{1}{\nu} E_{31} & \frac{1}{\nu j_{1}} E_{32} & Y
\end{array}\right)= \\
& =\left(\begin{array}{cc|c}
\nu j_{1}\left(E_{11}+\frac{1}{2} Y\right) & \nu E_{12} & -j_{1} E_{23} \\
\nu E_{21} & \nu j_{1}\left(-E_{22}+\frac{1}{2} Y\right) & -E_{23} \\
\hline j_{1} E_{31} & E_{32} & \nu j_{1} Y
\end{array}\right) .
\end{aligned}
$$

The first order Casimir operator disappear $C_{1}\left(j_{1}\right)=\operatorname{str} \bar{E}\left(j_{1}\right)=0$. The second order Casimir operator is as follows

$$
\begin{gathered}
C_{2}\left(j_{1}\right)=\operatorname{str}\left(\bar{E}\left(j_{1}\right)\right)^{2}=\nu^{2} j_{1}^{2}\left(2 E_{11}^{2}-\frac{1}{2} Y^{2}\right)+\nu^{2}\left(E_{12} E_{21}+E_{21} E_{12}\right)+ \\
+j_{1}^{2}\left(E_{31} E_{13}+E_{13} E_{31}\right)+E_{32} E_{23}-E_{23} E_{32} .
\end{gathered}
$$

In the case of superalgebras $\operatorname{osp}(M \mid N)$ the multiplier in (23) is equal to $\nu(1, M)\left[1, \frac{N}{2}\right]$ and all formulas for matrix $\bar{E}(j ; \epsilon)$ and matrix elements $(\bar{E}(j ; \epsilon))_{k p}$ appear as for the $s l(m ; j \mid n ; \epsilon)$ with substitution $m=M$ and 
$n=\frac{N}{2}$. Let us consider the $\operatorname{osp}(1 \mid 2 ; \nu)$ superalgebra as an example. Their generators are transformed as

$$
E_{12}=\nu E_{12}^{\star}, E_{13}=\nu E_{13}^{\star}, E_{23}=E_{23}^{\star}, E_{32}=E_{32}^{\star}, E_{22}=E_{22}^{\star},
$$

and matrix $\bar{E}(\nu)$ is given by

$$
\bar{E}(\nu)=-\nu\left(\begin{array}{c|cc}
0 & \frac{1}{\nu} E_{12} & \frac{1}{\nu} E_{13} \\
\hline \frac{1}{\nu} E_{13} & E_{22} & E_{23} \\
-\frac{1}{\nu} E_{12} & E_{32} & -E_{22}
\end{array}\right)=-\left(\begin{array}{c|cc}
0 & E_{12} & E_{13} \\
\hline E_{13} & \nu E_{22} & \nu E_{23} \\
-E_{12} & \nu E_{32} & -\nu E_{22}
\end{array}\right) .
$$

The first order Casimir operator is equal to zero $C_{1}(\nu)=\operatorname{str} \bar{E}(\nu)=0$ and the second order Casimir operator is represented as

$$
C_{2}(\nu)=\nu^{2} E_{22}^{2}+\left(E_{12} E_{13}-E_{13} E_{12}\right)-\frac{1}{2} \nu^{2}\left(E_{32} E_{23}+E_{23} E_{32}\right) .
$$

\section{Conclusion}

Using classical CK Lie algebras of different type we have built basic CK superalgebras. Unlike standard procedure [19] of zero tending parameter contractions in this work are described with the help of nilpotent valued parameters. Such approach gives an opportunity to obtain the distribution of contraction parameters among superalgebra generators starting from quadratic form and hence to build CK superalgebras by means of pure algebraic tools without limiting procedure. Contracted superalgebras are connected with transformations of superspaces with nilpotent cartesian coordinates and represent a wide class of different semidirect sums for different possible contractions. An infinite sequences of Casimir elements of CK superalgebras have been obtained by a suitable transformations of the standard expressions of the corresponding operators of the basic superalgebras. It is our hope that CK superalgebras will be relevant for construction of supersymmetric physical models.

\section{Acknowledgments}

This work was supported by Russian Foundation for Basic Research under Project 01-01-96433. 


\section{References}

[1] Golfand Yu A and Likhtman E P 1971 JETP. Lett.13 452

[2] Volkov D V and Akulov V P 1972 JETP Lett. 16621

[3] Wess J and Zumino B 1974 Nucl. Phys. B70 139

[4] West P 1986 Introduction to supersymmetry and supergravity, (Singapore: World Scientific)

[5] Keck B W 1975 J.Phys.A: Math.Gen. 81819

[6] Vasiliev M A 1990 Phys.Lett.B 243378

[7] Bars I 1996 Preprint hep-th/9608061

[8] Bellucci S, Ivanov E and Krivonos S 2001 Nucl.Phys.B (Proc. Suppl.) $102 \& 10326$

[9] Gromov N A 1990 Contractions and Analytical Continuations of Classical Groups. Unified Approach (Syktyvkar: Komi SC) (in Russian)

[10] Gromov N A and Man'ko V I 1990 J.Math.Phys. 31 1054, 1060

[11] Rembielinski J, Tybor W 1984 Acta Physica Polonica B15 611

[12] Hussin V, Negro J and del Olmo M A 1999 J.Phys.A: Math.Gen. 32 5097

[13] Patra M K and Tripathy K C 1989 Lett.Math.Phys 171

[14] Moody R V and Patera J 1991 J.Phys.A: Math.Gen.24 2227

[15] Gromov N A, Kostyakov I V and Kuratov V V Preprint arXiv:hepth/0110257

[16] Frappat L, Sciarrino A and Sorba P 1996 Dictionary on Lie Superalgebras (hep-th/9607161, ENSLAPP-AL-600/96 and DSF-T-30/96)

[17] Leites D and Sergeev A 2002 Preprint arXiv:math.RT/0202180 v1 
[18] Arnaudon D, Chryssomalakos C and Frappat L 1995 Preprint arXiv:qalg/9503021 v2, ENSLAPP-A-505/95

[19] Inönü E and Wigner E P 1953 Proc.Nat.Acad.Sci. USA 39510 